УДК 517.946

\title{
Ill-posed Boundary-value Problem for a System of Partial Differential Equations with Two Degenerate Lines
}

\author{
Kudratillo S. Fayazov* \\ Turin Polytechnic University in Tashkent \\ Kichik Khalka Yuli Str. 17, Tashkent, 100195 \\ Uzbekistan \\ Yashin K. Khudayberganov ${ }^{\dagger}$ \\ National University of Uzbekistan \\ Universitet Str. 4, Tashkent, 100174 \\ Uzbekistan
}

Received 20.09.2018, received in revised form 18.01.2019, accepted 26.03.2019

This paper is devoted to the investigation of ill-posed boundary-value problem for system of parabolic type equations with changing time direction with two degenerate lines. The problem under consideration is ill-posed in the sense of J. Hadamard, namely, there is no continuous dependence of the solution on the initial data. Such equations have many different applications, for example, describe the processes of heat propagation in inhomogeneous media, the interaction of filtration flows, mass transfer near the surface of an aircraft, and the description of complex viscous fluid flows. As possible applications should also indicate the task of calculating heat exchangers, in which the counter flow principle is used. Theorems on the uniqueness and conditional stability of a solution on a set of well-posedness are proved. We construct a sequence of approximate (regularized) solutions that are stable on the set of well-posedness.

Keywords: parabolic equation with changing time direction, ill-posed problem, a priori estimate, estimation of conditional stability, uniqueness of solution, approximate solution.

DOI: 10.17516/1997-1397-2019-12-3-392-401.

The paper is devoted to the investigation of ill-posed boundary-value problem for a system of partial differential equations with two degeneration lines.

\section{Introduction and preliminaries}

We consider the problem of finding a solution $(u(x, y, t), \nu(x, y, t))$ of the system of equations

$$
\left\{\begin{array}{l}
\left(\frac{\partial}{\partial t}-\operatorname{sign}(x) \frac{\partial}{\partial x^{2}}-\operatorname{sign}(y) \frac{\partial}{\partial y^{2}}\right) \nu(x, y, t)=0 \\
\left(\frac{\partial}{\partial t}-\operatorname{sign}(x) \frac{\partial}{\partial x^{2}}-\operatorname{sign}(y) \frac{\partial}{\partial y^{2}}\right) u(x, y, t)=\nu(x, y, t)
\end{array}\right.
$$

in the region $\Omega=\{(x, y, t) \mid(-1<x<1) \times(-1<y<1) \times(0<t<T<\infty), x \neq 0, y \neq 0\}$ that satisfy the following conditions:

initial conditions

$$
u(x, y, 0)=f(x, y), \nu(x, y, 0)=\varphi(x, y), \quad(x, y) \in \prod=\{-1 \leqslant x \leqslant 1, \quad-1 \leqslant y \leqslant 1\},
$$

\footnotetext{
${ }^{*}$ kudratillo52@mail.ru

${ }^{\dagger}$ komilyashin89@mail.ru

(c) Siberian Federal University. All rights reserved
} 
boundary conditions

$$
\begin{aligned}
& u(-1, y, t)=u(+1, y, t)=0, \quad-1 \leqslant y \leqslant 1,0 \leqslant t \leqslant T, \\
& u(x,-1, t)=u(x,+1, t)=0, \quad-1 \leqslant x \leqslant 1,0 \leqslant t \leqslant T, \\
& \nu(-1, y, t)=\nu(+1, y, t)=0, \quad-1 \leqslant y \leqslant 1,0 \leqslant t \leqslant T, \\
& \nu(x,-1, t)=\nu(x,+1, t)=0, \quad-1 \leqslant x \leqslant 1,0 \leqslant t \leqslant T,
\end{aligned}
$$

and gluing conditions

$$
\begin{array}{ll}
u(-0, y, t)=u(+0, y, t), & u_{x}(-0, y, t)=u_{x}(+0, y, t), \quad-1 \leqslant y \leqslant 1,0 \leqslant t \leqslant T, \\
u(x,-0, t)=u(x,+0, t), & u_{y}(x,-0, t)=u_{y}(x,+0, t), \quad-1 \leqslant x \leqslant 1,0 \leqslant t \leqslant T, \\
\nu(-0, y, t)=\nu(+0, y, t), & \nu_{x}(-0, y, t)=\nu_{x}(+0, y, t), \quad-1 \leqslant y \leqslant 1,0 \leqslant t \leqslant T, \\
\nu(x,-0, t)=\nu(x,+0, t), & \nu_{y}(x,-0, t)=\nu_{y}(x,+0, t), \quad-1 \leqslant x \leqslant 1,0 \leqslant t \leqslant T .
\end{array}
$$

In many cases, when solving applied problems one uses the methods of mathematical physics. By solving theoretical and practical problems, one can often meet mixed type equations, which are one of the main objects of the chapters of the theory of modern differential equations. In this paper we consider an ill-posed (in the sense of J. Hadamard) boundary-value problem for a system of parabolic type equations with a changing time direction.

For parabolic type equations with changing time directions problems were first considered by M. Jevrey [1]. Various boundary value problems for such equations have been the subject of research by several scientists, such as S. D. Pagani [2], G. Talenti, S. A. Tersenov [3], V. N. Vragov [4], V. K. Romanko [5], A. M. Nakhushev [6] and their students. To study these problems, in these papers such classical methods as various analogues of the Green's function, potential theory, integral equations were used. In the work of S. A. Tersenov [3] the results of research using classical methods are summed up.

From further research it became clear that parabolic equations with a changing time direction can be considered as a special case of equations of a mixed type. In the works of N. V. Kislov [7], the generalized solvability of boundary value problems for abstract type equation was proved. A great contribution to the study of boundary value problems for equations of mixed type was made by A. I. Kozhanov [8], I. E. Egorov [9], S. G. Pyatkov [10, 11], A. A. Kerefov [12], I. S. Pulkin [13], K. B. Sabitov [14], and others.

The problem under investigation belongs to the class of ill-posed problems in mathematical physics, namely, in this problem there is no continuous dependence of the solution on the initial data. Ill-posed problems for such equations was considered in the works of H. Levine, A. L. Buchkheim, K.S Fayazov [15], K.S. Fayazov and I. O. Khazhiev [16], K. S. Fayazov and Y. K. Khudayberganov [17] and others.

Such equations have many different applications, for example, the description the processes of heat propagation in inhomogeneous media, the interaction of filtration flows, mass transfer near the surface of an aircraft, and the description of complex viscous fluid flows. As possible applications should also indicate the problem of calculating heat exchangers, in which the principle of counter flow is used (see $[3,18]$ ).

In the previous works were examined correct and incorrect problems for the classic and mixed-type equations with one degenerate lines. Correct problems for Laplas equation with two degenerate lines was considered by A. A. Gamaltdinova in the work [19]. In this paper, based on the idea of A. N. Tikhonov, the conditional correctness of problem (1)-(4) is investigate, namely, theorems on uniqueness and conditional stability were proved, and approximate solutions that are stable on the set of well-posedness are constructed. 


\section{Spectral problem}

Find the values of $\lambda$ for which the following problem

$\operatorname{sign}(x) \vartheta_{x x}(x, y)+\operatorname{sign}(y) \vartheta_{y y}(x, y)=-\lambda \vartheta(x, y),-1<x<1,-1<y<1, x \neq 0, y \neq 0$,

$$
\begin{gathered}
\vartheta(-1, y)=\vartheta(+1, y)=0, \quad-1 \leqslant y \leqslant 1, \\
\vartheta(-0, y)=\vartheta(+0, y), \quad \vartheta_{x}(-0, y)=\vartheta_{x}(+0, y), \quad-1 \leqslant y \leqslant 1, \\
\vartheta(x,-1)=\vartheta(x,+1)=0, \quad-1 \leqslant x \leqslant 1, \\
\vartheta(x,-0)=\vartheta(x,+0), \quad \vartheta_{y}(x,-0)=\vartheta_{y}(x,+0), \quad-1 \leqslant x \leqslant 1,
\end{gathered}
$$

has nontrivial solution.

According to the result of [11], this problem (5)-(6) has a no decreasing sequence of $\lambda_{k, l}^{++}, \lambda_{k, l}^{+-}, \lambda_{k, l}^{-+}, \lambda_{k, l}^{--}$eigenvalues and the corresponding Eigne functions $\left\{\vartheta_{k, l}^{++}\right\}_{k, l=1}^{\infty}$, $\left\{\vartheta_{k, l}^{+-}\right\}_{k, l=1}^{\infty},\left\{\vartheta_{k, l}^{-+}\right\}_{k, l=1}^{\infty},\left\{\vartheta_{k, l}^{--}\right\}_{k, l=1}^{\infty}$ (see also [17]).

Let $\|u\|^{2}=(u, u)$ be the scalar product of $(u, v)=\int_{-1}^{1} \int_{-1}^{1} u v d x d y$. Besides

$$
\begin{gathered}
\left(\operatorname{sign}(x) \operatorname{sign}(y) \vartheta_{k, l}^{ \pm \pm}, \vartheta_{i, j}^{ \pm \pm}\right)=\delta_{k, i} \cdot \delta_{l, j}, \\
\left(\operatorname{sign}(x) \operatorname{sign}(y) \vartheta_{k, l}^{ \pm \mp}, \vartheta_{i, j}^{ \pm \mp}\right)=-\delta_{k, i} \cdot \delta_{l, j}, \delta_{k, i}=\left\{\begin{array}{l}
1, k=i, \\
0, k \neq i,
\end{array}\right. \\
\left(\operatorname{sign}(x) \operatorname{sign}(y) \vartheta_{k l}^{ \pm \mp}, \vartheta_{i j}^{\mp \pm}\right)=0,
\end{gathered}
$$

where $k, l, i, j=1,2,3, \ldots$.

We represent the spectral projection $-P^{ \pm}$in the following form

$$
\begin{aligned}
& P^{+} \phi=\sum_{k, l=1}^{\infty}\left(\operatorname{sign}(x) \operatorname{sign}(y) \phi, \vartheta_{k, l}^{++}\right) \vartheta_{k, l}^{++}+\left(\operatorname{sign}(x) \operatorname{sign}(y) \phi, \vartheta_{k, l}^{--}\right) \vartheta_{k, l}^{--}, \\
& P^{-} \phi=\sum_{k, l=1}^{\infty}\left(\operatorname{sign}(x) \operatorname{sign}(y) \phi, \vartheta_{k, l}^{+-}\right) \vartheta_{k, l}^{+-}+\left(\operatorname{sign}(x) \operatorname{sign}(y) \phi, \vartheta_{k, l}^{-+}\right) \vartheta_{k, l}^{-+} .
\end{aligned}
$$

See $[10]$

$$
\begin{gathered}
\left(P^{+}-P^{-}\right) \phi=\phi, \quad\left(\operatorname{sign}(x) \operatorname{sign}(y)\left(P^{+}-P^{-}\right) \phi, \phi\right)=\|\phi\|^{2}, \\
\left(\operatorname{sign}(x) \operatorname{sign}(y) P^{ \pm} \phi, \psi\right)=\left(\operatorname{sign}(x) \operatorname{sign}(y) \phi, P^{ \pm} \psi\right), \quad \phi, \psi \in \mathrm{H}_{0}=L_{2}(-1,1) \times(-1,1), \\
\|\vartheta\|_{0}^{2}=\sum_{k, l=1}^{\infty}\left\{\left|\left(\operatorname{sign}(x) \operatorname{sign}(y) \vartheta, \vartheta_{k, l}^{++}\right)\right|^{2}+\left|\left(\operatorname{sign}(x) \operatorname{sign}(y) \vartheta, \vartheta_{k, l}^{+-}\right)\right|^{2}+\right. \\
\left.+\left|\left(\operatorname{sign}(x) \operatorname{sign}(y) \vartheta, \vartheta_{k, l}^{-+}\right)\right|^{2}+\left|\left(\operatorname{sign}(x) \operatorname{sign}(y) \vartheta, \vartheta_{k, l}^{-}\right)\right|^{2}\right\} .
\end{gathered}
$$

According to the results of [11], the Eigen functions of problem (5)-(6) form a Rises basis. 


\section{A priori estimate}

Definition 3.1. By the solution of the problem we mean a pair of functions $(u(x, y, t), \nu(x, y, t))$ having continuous derivatives entering to the equation that satisfies the system of equations (1) and conditions (2)-(4).

Lemma 3.1. Let the function $\nu(x, y, t)$ satisfy the equation $\nu_{t}(x, y, t)=\operatorname{sign}(x) \nu_{x x}(x, y, t)+$ $+\operatorname{sign}(y) \nu_{y y}(x, y, t)$ in the domain $\Omega$ and conditions

$$
\begin{aligned}
& \nu(-1, y, t)=\nu(+1, y, t)=0, \quad \nu(-0, y, t)=\nu(+0, y, t), \quad \nu_{x}(-0, y, t)=\nu_{x}(+0, y, t), \\
& \nu(x,-1, t)=\nu(x,+1, t)=0, \quad \nu(x,-0, t)=\nu(x,+0, t), \quad \nu_{y}(x,-0, t)=\nu_{y}(x,+0, t) .
\end{aligned}
$$

Then for $\nu(x, y, t)$ we have the estimate

$$
\|\nu(x, y, t)\|_{0} \leqslant \sqrt{2}\|\nu(x, y, 0)\|_{0}^{\frac{T-t}{T}} \cdot\|\nu(x, y, T)\|_{0}^{\frac{t}{T}} .
$$

Proof of this Lemma 3.1 you can find in [17] Theorem 3.1.

Remark. For the easy presentation without limiting generality, we assume that in the expansion of functions $f(x, y)$ and $\varphi(x, y)$ in series of the eigenfunctions of the spectral problem, the coefficients in front of the eigenfunctions $\vartheta_{k, l}^{+-}$and $\vartheta_{k, l}^{-+}$for any $k, l \in N$ are identically zero.

Lemma 3.2. Let $u(x, y, t)$ be a function satisfying equation

$$
u_{t}(x, y, t)=\operatorname{sign}(x) u_{x x}(x, y, t)+\operatorname{sign}(y) u_{y y}(x, y, t)+\nu(x, y, t)
$$

in the region $\Omega$ and the conditions:

$$
\begin{array}{ll}
u(-1, y, t)=u(+1, y, t)=0, & u(x,-1, t)=u(x,+1, t)=0, \\
u(-0, y, t)=u(+0, y, t), & u(x,-0, t)=u(x,+0, t), \\
u_{x}(-0, y, t)=u_{x}(+0, y, t), & u_{y}(x,-0, t)=u_{y}(x,+0, t) .
\end{array}
$$

Then for $u(x, y, t)$ the following inequality holds

$$
\|u(x, y, t)\|_{0} \leqslant \sqrt{2}\left(\|u(x, y, 0)\|_{0}+\|\gamma\|_{0}\right)^{\frac{T-t}{T}} \cdot\left(\|u(x, y, T)\|_{0}+\|\gamma\|_{0}\right)^{\frac{t}{T}}+\|\gamma\|_{0},
$$

where $\|\gamma\|_{0}=\left(\int_{0}^{T}\|\nu(x, y, t)\|_{0}^{2} d t\right)^{\frac{1}{2}}$.

Proof. We present the solutions of (7) in the form

$$
u(x, y, t)=\varpi(x, y, t)+\omega(x, y, t)
$$

here $\omega(x, y, t)$ is the solution of the homogeneous equation

$$
\omega_{t}(x, y, t)=\operatorname{sign}(x) \omega_{x x}(x, y, t)+\operatorname{sign}(y) \omega_{y y}(x, y, t),
$$

and $\varpi(x, y, t)$ is a solution of a non-homogeneous equation.

$$
\varpi_{t}(x, y, t)=\operatorname{sign}(x) \varpi_{x x}(x, y, t)+\operatorname{sign}(y) \varpi_{y y}(x, y, t)+\nu(x, y, t) .
$$

We introduce the notation $\lambda_{k, l}^{ \pm \pm}=\lambda_{k, l}^{ \pm}, \quad \vartheta_{k, l}^{ \pm \pm}=\vartheta_{k, l}^{ \pm}$. 
The functions $\omega(x, y, t), \varpi(x, y, t)$ satisfy conditions $(8)$. We represent the solution of problems $(9-10)$ in the form

$$
\begin{gathered}
\omega(x, y, t)=\sum_{k, l=1}^{\infty} \omega_{k, l}^{+}(t) \vartheta_{k, l}^{+}+\sum_{k, l=1}^{\infty} \omega_{k, l}^{-}(t) \vartheta_{k, l}^{-}, \\
\varpi(x, y, t)=\sum_{k, l=1}^{\infty} \varpi_{k, l}^{+}(t) \vartheta_{k, l}^{+}+\sum_{k, l=1}^{\infty} \varpi_{k, l}^{-}(t) \vartheta_{k, l}^{-},
\end{gathered}
$$

here the functions $\omega_{k, l}^{ \pm}(t), \varpi_{k, l}^{ \pm}(t)$ are solutions of the following problems

$$
\begin{aligned}
& \left\{\begin{array} { l } 
{ ( \varpi _ { k , l } ^ { + } ( t ) ) _ { t } + \lambda _ { k , l } ^ { + } \varpi _ { k , l } ^ { + } ( t ) = \nu _ { k , l } ^ { + } ( t ) , } \\
{ \varpi _ { k , l } ^ { + } ( 0 ) = 0 , }
\end{array} \quad \left\{\begin{array}{l}
\left(\varpi_{k, l}^{-}(t)\right)_{t}+\lambda_{k, l}^{-} \varpi_{k, l}^{-}(t)=\nu_{k, l}^{-}(t), \\
\varpi_{k, l}^{-}(0)=-\int_{0}^{T} e^{\lambda_{k, l}^{-} \tau} \nu_{k, l}^{-}(\tau) d \tau,
\end{array}\right.\right. \\
& \left\{\begin{array} { l } 
{ ( \omega _ { k , l } ^ { + } ( t ) ) _ { t } + \lambda _ { k , l } ^ { + } \omega _ { k , l } ^ { + } ( t ) = 0 , } \\
{ \omega _ { k , l } ^ { + } ( 0 ) = f _ { k , l } ^ { + } , }
\end{array} \left\{\begin{array}{l}
\left(\omega_{k, l}^{-}(t)\right)_{t}+\lambda_{k, l}^{-} \omega_{k, l}^{-}(t)=0 \\
\omega_{k, l}^{-}(0)=f_{k, l}^{-}+\int_{0}^{T} e^{\lambda_{k, l}^{-} \tau} \nu_{k, l}^{-}(\tau) d \tau
\end{array}\right.\right.
\end{aligned}
$$

where

$$
\begin{aligned}
\nu_{k, l}^{ \pm}(t) & = \pm \int_{-1}^{1} \int_{-1}^{1} \operatorname{sign}(x) \operatorname{sign}(y) \nu(x, y, t) \vartheta_{k, l}^{ \pm}(x, y) d x d y \\
f_{k, l}^{ \pm}(t) & = \pm \int_{-1}^{1} \int_{-1}^{1} \operatorname{sign}(x) \operatorname{sign}(y) f(x, y) \vartheta_{k, l}^{ \pm}(x, y) d x d y
\end{aligned}
$$

It is not difficult to see that [20]

$$
\varpi_{k, l}^{+}(t)=\int_{0}^{t} e^{\lambda_{k, l}^{+}(\tau-t)} \nu_{k, l}^{+}(\tau) d \tau, \quad \varpi_{k, l}^{-}(t)=-\int_{t}^{T} e^{\lambda_{k, l}^{-}(\tau-t)} \nu_{k, l}^{-}(\tau) d \tau,
$$

and

$$
\|\varpi(x, y, t)\|_{0}^{2}=\sum_{k, l=1}^{\infty}\left\{\varpi_{k, l}^{+}(t)\right\}^{2}+\sum_{k, l=1}^{\infty}\left\{\varpi_{k, l}^{-}(t)\right\}^{2} \leqslant \int_{0}^{T}\|\nu(x, y, t)\|_{0}^{2} d t .
$$

The solution of the equation (11) can be presented as

$$
\omega_{k, l}^{+}(t)=f_{k, l}^{+} e^{-\lambda_{k, l}^{+} t}, \omega_{k, l}^{-}(t)=f_{k, l}^{-} e^{-\lambda_{k, l}^{-} t}+\int_{0}^{T} e^{\lambda_{k, l}^{-}(\tau-t)} \nu_{k, l}^{-}(\tau) d \tau .
$$

According to Lemma 3.1 for the solution $\omega(x, y, t)$ the following estimate holds

$$
\|\omega(x, y, t)\|_{0} \leqslant \sqrt{2}\left(\|\omega(x, y, 0)\|_{0}\right)^{\frac{T-t}{T}} \cdot\left(\|\omega(x, y, T)\|_{0}\right)^{\frac{t}{T}} .
$$

Therefore, for the functions $u(x, y, t)=\omega(x, y, t)+\varpi(x, y, t)$, we obtain the estimate

$$
\|u(x, y, t)\|_{0} \leqslant \sqrt{2}\left(\|u(x, y, 0)\|_{0}+\|\gamma\|_{0}\right)^{\frac{T-t}{T}} \cdot\left(\|u(x, y, T)\|_{0}+\|\gamma\|_{0}\right)^{\frac{t}{T}}+\|\gamma\|_{0},
$$

where $\|\gamma\|_{0}=\left(\int_{0}^{T}\|\nu(x, y, t)\|_{0}^{2} d t\right)^{\frac{1}{2}}$. 


\section{Uniqueness and conditional stability}

Let

$$
M=\left\{(u, \nu):\|u(x, y, T)\|_{0}+\|\nu(x, y, T)\|_{0} \leqslant m, m<\infty\right\}
$$

be the set of correctness of problem (1)-(4).

Theorem 4.1. Let the solution of problem (1)-(4) exist and $(u(x, y, t), \nu(x, y, t))$. Then the solution of problem (1)-(4) is unique.

Proof. Let the pairs of functions $\left(u_{1}(x, y, t), \nu_{1}(x, y, t)\right),\left(u_{2}(x, y, t), \nu_{2}(x, y, t)\right)$ be solutions of problem (1)-(4). We introduce the notation $u(x, y, t)=u_{1}(x, y, t)-u_{2}(x, y, t)$ and $\nu(x, y, t)=$ $=\nu_{1}(x, y, t)-\nu_{2}(x, y, t)$ then the pair of functions $(u(x, y, t), \nu(x, y, t)) \in M$. satisfies the problem (1)-(4) with $u(x, y, 0)=0, \nu(x, y, 0)=0$. By Lemma 3.1 and Lemma 3.2, we have $\|\nu(x, y, t)\|_{0}=$ $=0$ and $\|u(x, y, t)\|_{0}=0$. Hence for any $(x, y, t) \in \Omega$ is true $u_{1}(x, y, t) \equiv u_{2}(x, y, t), \nu_{1}(x, y, t) \equiv$ $\nu_{2}(x, y, t)$. The Theorem 4.1 proved.

Let $U(x, y, t)=u(x, y, t)-u_{\varepsilon}(x, y, t), \quad V(x, y, t)=\nu(x, y, t)-\nu_{\varepsilon}(x, y, t)$, where $(u(x, y, t), \nu(x, y, t))$ is solution of problem (1)-(4) with exact data, $\left(u_{\varepsilon}(x, y, t), \nu_{\varepsilon}(x, y, t)\right)$ is solution of problem (1)-(4) with approximate data.

Theorem 4.2. Let $(u(x, y, t), \nu(x, y, t)) \in M,\left(u_{\varepsilon}(x, y, t), \nu_{\varepsilon}(x, y, t)\right) \in M \quad$ and $\left\|\varphi(x, y)-\varphi_{\varepsilon}(x, y)\right\|_{0} \leqslant \varepsilon, \quad\left\|f(x, y)-f_{\varepsilon}(x, y)\right\|_{0} \leqslant \varepsilon$. Then by any $t \in[0 ; T]$ for $(U, V)$ the estimates

$$
\begin{gathered}
\|V(x, y, t)\|_{0} \leqslant \sqrt{2}(\varepsilon)^{1-\frac{t}{T}}(2 m)^{\frac{t}{T}}, \\
\|U(x, y, t)\|_{0} \leqslant\left(\varepsilon+\left\|\gamma_{\varepsilon}\right\|_{0}\right)^{1-\frac{t}{T}}\left(2 m+\left\|\gamma_{\varepsilon}\right\|_{0}\right)^{\frac{t}{T}}+\left\|\gamma_{\varepsilon}\right\|_{0},
\end{gathered}
$$

hold, where $\left\|\gamma_{\varepsilon}\right\|_{0}=\left(\int_{0}^{T} 2\left(\varepsilon^{2}\right)^{1-\frac{t}{T}}\left(4 m^{2}\right)^{\frac{t}{T}} d t\right)^{\frac{1}{2}}$.

Proof. The pair of functions $(U(x, y, t), V(x, y, t))$ satisfies conditions (1)-(4), where

$$
\begin{aligned}
& U(x, y, 0)=f(x, y)-f_{\varepsilon}(x, y), \\
& V(x, y, 0)=\varphi(x, y)-\varphi_{\varepsilon}(x, y), \quad-1 \leqslant x \leqslant 1, \quad-1 \leqslant y \leqslant 1 .
\end{aligned}
$$

According to the conditions of Theorem

$$
\begin{gathered}
\|V(x, y, 0)\|_{0}=\left\|\varphi(x, y)-\varphi_{\varepsilon}(x, y)\right\|_{0} \leqslant \varepsilon, \quad \| V\left(x, y, T \|_{0} \leqslant 2 m,\right. \\
\|U(x, y, 0)\|_{0}=\left\|f(x, y)-f_{\varepsilon}(x, y)\right\|_{0} \leqslant \varepsilon, \quad \| U\left(x, y, T \|_{0} \leqslant 2 m .\right.
\end{gathered}
$$

Then from Lemma 3.1 and Lemma 3.2 it yields

$$
\begin{gathered}
\|V(x, y, t)\|_{0} \leqslant \sqrt{2}(\varepsilon)^{1-\frac{t}{T}}(2 m)^{\frac{t}{T}}, \\
\|U(x, y, t)\|_{0} \leqslant\left(\varepsilon+\left\|\gamma_{\varepsilon}\right\|_{0}\right)^{1-\frac{t}{T}}\left(2 m+\left\|\gamma_{\varepsilon}\right\|_{0}\right)^{\frac{t}{T}}+\left\|\gamma_{\varepsilon}\right\|_{0} .
\end{gathered}
$$

The theorem is proved. 


\section{Approximate solution}

Let in the problem (1)-(4) $f(x, y)=0$. Then the solution $(u(x, y, t), \nu(x, y, t))$ can be presented in the form

$$
\begin{aligned}
& \nu(x, y, t)=\sum_{k, l=1}^{\infty} \varphi_{k, l}^{+} e^{-\lambda_{k, l}^{+} t} \vartheta_{k, l}^{+}+\sum_{k, l=1}^{\infty} \varphi_{k, l}^{-} e^{-\lambda_{k, l}^{-}} \vartheta_{k, l}^{-}, \\
& u(x, y, t)=\sum_{k, l=1}^{\infty} \varphi_{k, l}^{+} t e^{-\lambda_{k, l}^{+} t} \vartheta_{k, l}^{+}+\sum_{k, l=1}^{\infty} \varphi_{k, l}^{-} t e^{-\lambda_{k, l}^{-} t} \vartheta_{k, l}^{-},
\end{aligned}
$$

where $\varphi_{k, l}^{ \pm}= \pm \int_{-1}^{1} \int_{-1}^{1} \operatorname{sign}(x) \operatorname{sign}(y) \varphi(x, y) \vartheta_{k, l}^{ \pm}(x, y) d x d y$.

An approximate solution of the problem is as a sequence of functions given below

$$
\begin{aligned}
& \nu^{N}(x, y, t)=\sum_{k, l=1}^{N} \varphi_{k, l}^{+} e^{-\lambda_{k, l}^{+} t} \vartheta_{k, l}^{+}+\sum_{k, l=1}^{N} \varphi_{k, l}^{-} e^{-\lambda_{k, l}^{-} t} \vartheta_{k, l}^{-}, \\
& u^{N}(x, y, t)=\sum_{k, l=1}^{N} \varphi_{k, l}^{+} t e^{-\lambda_{k, l}^{+} t} \vartheta_{k, l}^{+}+\sum_{k, l=1}^{N} \varphi_{k, l}^{-} t e^{-\lambda_{k, l}^{-}} \vartheta_{k, l}^{-},
\end{aligned}
$$

here $N$ is the integer number of the parameter regularization. An approximate solution with approximate data has the form

$$
\begin{aligned}
& \nu_{\varepsilon}^{N}(x, y, t)=\sum_{k, l=1}^{N} \varphi_{\varepsilon_{k, l}^{+}}^{+} e^{-\lambda_{k, l}^{+} t} \vartheta_{k, l}^{+}+\sum_{k, l=1}^{N} \varphi_{\varepsilon}^{-} \bar{\varepsilon}_{k, l} e^{-\lambda_{k, l}^{-} t} \vartheta_{k, l}^{-}, \\
& u_{\varepsilon}^{N}(x, y, t)=\sum_{k, l=1}^{N} \varphi_{\varepsilon_{k, l}^{+}}^{+} t e^{-\lambda_{k, l}^{+} t} \vartheta_{k, l}^{+}+\sum_{k, l=1}^{N} \varphi_{\varepsilon}^{-} t e^{-\lambda_{k, l}^{-} t} \vartheta_{k, l}^{-},
\end{aligned}
$$

Let $\left\|\varphi(x, y)-\varphi_{\varepsilon}(x, y)\right\|_{0} \leqslant \varepsilon$ and $(u(x, y, t), \nu(x, y, t)) \in M$. Then we estimate the norm of the difference between the exact and approximate solution

$$
\begin{aligned}
\left\|\nu(x, y, t)-\nu_{\varepsilon}^{N}(x, y, t)\right\|_{0} & =\left\|\nu(x, y, t)-\nu^{N}(x, y, t)+\nu^{N}(x, y, t)-\nu_{\varepsilon}^{N}(x, y, t)\right\|_{0} \leqslant \\
& \leqslant\left\|\nu(x, y, t)-\nu^{N}(x, y, t)\right\|_{0}+\left\|\nu^{N}(x, y, t)-\nu_{\varepsilon}^{N}(x, y, t)\right\|_{0}, \\
\left\|u(x, y, t)-u_{\varepsilon}^{N}(x, y, t)\right\|_{0} & =\left\|u(x, y, t)-u^{N}(x, y, t)+u^{N}(x, y, t)-u_{\varepsilon}^{N}(x, y, t)\right\|_{0} \leqslant \\
& \leqslant\left\|u(x, y, t)-u^{N}(x, y, t)\right\|_{0}+\left\|u^{N}(x, y, t)-u_{\varepsilon}^{N}(x, y, t)\right\|_{0} .
\end{aligned}
$$

Estimating the second term on the right-hand side of (12) we get

$$
\begin{aligned}
\| \nu^{N}(x, y, t) & -\nu_{\varepsilon}^{N}(x, y, t) \|_{0}^{2}=\sum_{k, l=1}^{N} e^{-2 \lambda_{k, l}^{+} t}\left(\varphi_{k, l}^{+}-\varphi_{\varepsilon k, l}^{+}\right)^{2}+\sum_{k, l=1}^{N} e^{-2 \lambda_{k, l}^{-} t}\left(\varphi_{k, l}^{-}-\varphi_{\varepsilon k, l}^{-}\right)^{2} \leqslant \\
& \leqslant e^{-2 \lambda_{N, N}^{-} t} \sum_{k, l=1}^{N}\left(\left(\varphi_{k, l}^{-}-\varphi_{\varepsilon k, l}^{-}\right)^{2}+\left(\varphi_{k, l}^{+}-\varphi_{\varepsilon k}^{+}\right)^{2}\right) \leqslant e^{-2 \lambda_{N, N}^{-} t} \varepsilon^{2},
\end{aligned}
$$

or

$$
\left\|\nu^{N}(x, y, t)-\nu_{\varepsilon}^{N}(x, y, t)\right\|_{0} \leqslant e^{-\lambda_{N, N}^{-} t} \varepsilon
$$


We estimate the first term on the right-hand side of (12)

$$
\left\|\nu(x, y, t)-\nu^{N}(x, y, t)\right\|_{0}^{2}=\sum_{k, l=N+1}^{\infty}\left(e^{-2 \lambda_{k, l}^{+} t}\left\{\varphi_{k, l}^{+}\right\}^{2}+e^{-2 \lambda_{k, l}^{-} t}\left\{\varphi_{k, l}^{-}\right\}^{2}\right)
$$

on condition

$$
\sum_{k, l=N+1}^{\infty} e^{-2 \lambda_{k, l}^{-} T}\left\{\varphi_{k, l}^{-}\right\}^{2} \leqslant m^{2}
$$

We estimate expression (13) under the condition (14) by the method of Lagrange multipliers. One can see

$$
\varphi_{k, l}^{-}=\left\{\begin{array}{l}
0, \quad k, l \neq N+1 \\
e^{\lambda_{k, l}^{-} T} m, \quad k, l=N+1
\end{array}\right.
$$

As a result, we have

$$
\sum_{k, l=N+1}^{\infty} e^{-2 \lambda_{k, l}^{-} t}\left\{\varphi_{k, l}^{-}\right\}^{2} \leqslant m^{2} e^{2 \lambda_{N+1, N+1}^{-}(T-t)}
$$

Let's assume that the series $\sum_{k, l=1}^{\infty}\left(\left\{\varphi_{k, l}^{+}\right\}^{2}+\left\{\varphi_{k, l}^{-}\right\}^{2}\right)$ converges. Then it is not difficult to see

$$
\sum_{k, l=N+1}^{\infty} e^{-2 \lambda_{k, l}^{+} t}\left\{\varphi_{k, l}^{+}\right\}^{2} \leqslant \sum_{k, l=1}^{\infty}\left\{\varphi_{k, l}^{+}\right\}^{2}=\alpha(N)
$$

where $\alpha(N) \rightarrow 0$ for $N \rightarrow \infty$. Thus we have

$$
\left\|\nu(x, y, t)-\nu^{N}(x, y, t)\right\|_{0}^{2} \leqslant m^{2} e^{2 \lambda_{N+1, N+1}^{-}(T-t)}+\alpha(N) .
$$

Summing the above estimates, we have

$$
0.5\left\|\nu(x, y, t)-\nu_{\varepsilon}^{N}(x, y, t)\right\|_{0}^{2} \leqslant \varepsilon^{2} e^{-2 \lambda_{N, N}^{-} t}+m^{2} e^{2 \lambda_{N+1, N+1}^{-}(T-t)}+\alpha(N) .
$$

Similarly for $u(x, y, t)$ we have

$$
0.5\left\|u(x, y, t)-u_{\varepsilon}^{N}(x, y, t)\right\|_{0}^{2} \leqslant t^{2} e^{-2 \lambda_{N, N}^{-} t} \varepsilon^{2}+m^{2}\left(\frac{t}{T}\right)^{2} e^{2 \lambda_{N+1, N+1}^{-}(T-t)}+t^{2} \alpha(N) .
$$

Minimizing the right-hand side of (15) and (16) with respect to $N$,we find the corresponding regularization parameter. $m$ is selected depending from the application problem.

\section{References}

[1] M.Gevrey, Sur les equations aux derivees partielles du type parabolique, J. Math. Appl., (1914), Ch. 4, 105-137.

[2] C.D.Pagani, G.Talenti, On a forward-backward differential equation, Annali di Matematica pura et Applicata, 90(1971), M, 1-58.

[3] S.A.Tersenov, Parabolic equations with a varying direction of time, Novosibirsk, 1985 (in Russian). 
[4] V.N.Vragov, A.G.Podgaev, On well-posed problems for some equations of variable type, Sov. Math., Dokl., 24(1981), 253-257.

[5] V.K.Romanko, Eigenvalues of boundary value problems for certain equations which change type, Differentsialnye Uravneniya, 19(1983), no. 10, 1759-1764 (in Russian).

[6] A.M.Nakhushev, On a class of linear boundary value problems for hyperbolic and mixed types of second-order equations, Nalchik, Elbrus, 1992 (in Russian).

[7] N.V.Kislov, Nonhomogeneous boundary value problems for differential-operator equations of mixed type, and their application, Math. USSR-Sb., 531984), no. 1, 17-35.

[8] A.I.Kozhanov, Composite Type Equations and Inverse Problems, VSP, Utrecht, The Netherlands, 1999.

[9] I.E.Egorov, V.E.Fedorov, I.M.Tikhonova, Modified Galerkin method for the second order equation of mixed type and estimate of its error, Programming \& Computer Software, Bulletin SUSU MMCS, 9(2016), no. 4, 30-39 (in Russian).

[10] S.G.Pyatkov, Solvability of boundary value problems for a second-order mixed type equation, Neoclassical differential equations in partial derivatives, Sat. sci. tr. AN SSSR, Sib. Institute of Mathematics, Novosibirsk, 1988, 77-90 (in Russian).

[11] S.G.Pyatkov, Properties of Eigen functions of a certain spectral problem and their applications, Some Applications of Functional Analysis to Equations of Mathematical Physics, Inst. Mat., Novosibirsk, 1986, 65-84 (in Russian).

[12] A.A.Kerefov, The Gevrey problem for a mixed-parabolic equation, Differ. Uravn., 13(1977), no. 1, 76-83 (in Russian).

[13] I.S.Pulkin, Gevrey problem for parabolic equations with changing time direction, Electronic Journal of Differential Equations, (2006), no. 50, 1-9 (in Russian).

[14] K.B.Sabitov, On the theory of the Frankl problem for equations of mixed type, Izv. Math., 81(2017), no. 1, 99-136.

[15] K.S.Fayazov, An ill-posed boundary-value problem for a second order mixed type equation, Uzbekskiy Matematicheskiy Zhurnal, (1995), no. 2, 89-93 (in Russian).

[16] K.S.Fayazov, I.O.Khazhiev, An incorrect initial-boundary value problem for a system of equations of parabolic type with a changing time direction, Computational technologies, $R A S, \mathbf{2 2}(2017)$, no. 3, 103-114 (in Russian).

[17] K.S.Fayazov, Y.K.Xudayberganov, Ill-posed boundary value problem for a mixed type equation with two degenerate lines, Uzbek Mathematical Journal, (2018), no. 2, 32-42.

[18] F.I.Frankl, On the theory of Laval nozzles, Izv. AN SSSR. Ser. Mat., 6(1945), 387-422 (in Russian).

[19] A.A.Gimaltdinova, The Dirichlet problem for the Lavrent'ev-Bitsadze equation by two lines of type changing type in a rectangular domain, Doklady Mathematics , 91(2015), no. 1, $41-46$. 
[20] M.M.Lavrentev, L.Y.Saveliev, Theory of operators and ill-posed problems, Publishing House of the Institute of Mathematics, Novosibirsk, 2010 (in Russian).

\title{
Некорректная краевая задача для системы уравнений в частных производных с двумя линиями вырождения
}

Кудратилло С. Фаязов

Туринский политехнический университет в Ташкенте

Кичик Халка Йули, 17, Ташкент, 100195

Узбекистан

Яшин К. Худайберганов

Национальный университет Узбекистана

Университетская, 4, Ташкент, 100174

Узбекистан

\begin{abstract}
Данная работа посвящена исследованию некорректной краевой задачи для системы уравнений параболического типа с меняюшимся направлением времени с двумя линиями вырождения. Рассматриваемая задача некорректна по Ж. Адамару, а именно отсутствует непрерывная зависимость решения от данных задачи. Подобные уравнения имеют множество различных применений, например, описывают прочессы распространения тепла в неоднородных средах, взаимодействия фильтрачионных потоков, массопереноса вблизи поверхности летательного аппарата сложных течений вязкой жидкости. В качестве возможных приложений следует также указать задачи расчета теплообменников, в которых использутся принцип противотока. Доказаны теоремы о единственности и условной устойчивости решения на множестве корректности. Построена последовательность приближенных (регуляризованных) решений, устойчивых на множестве корректности.
\end{abstract}

Ключевые слова: параболическое уравнение с меняющимся направлением времени, некорректная задача, априорная оценка, оценка условной устойчивости, единственность решения, приближенное решение. 